\title{
Caracterização das mães, partos e recém-nascidos em Campinas, São Paulo, 2001 e 2005
}

\author{
Characterization of mothers, mode of deliveries and newborns in Campinas, São Paulo, 2001 and 2005
}

André Moreno Morcillo ${ }^{1}$, Emília de Faria Carniel ${ }^{2}$, Maria de Lurdes Zanolli ${ }^{3}$, Luisa Zanolli Moreno ${ }^{4}$, Maria Ângela R. de G. M. Antonio 3

RESUMO

Objetivo: Comparar dados pré-natais, dos partos e dos recém-nascidos de Campinas em 2001 e 2005.

Métodos: Estudo transversal que analisou 13.656 documentos do Sistema de Informações sobre Nascidos Vivos (Sinasc) de 2005, comparando-as aos resultados de 2001. Analisou-se o local de moradia e parto, idade materna, estado civil, escolaridade, ocupação, paridade, consultas de pré-natal, tipo de parto, duração da gestação e peso ao nascer. Para avaliar a associação entre as variáveis, utilizou-se o teste de qui-quadrado, sendo significante $p<0,05$.

Resultados: Os nascidos nos Distritos de Saúde (DS) com piores índices de condições de vida (ICV) diminuíram em relação a 2001. A taxa de adolescentes passou de 17,7\% para 14,7\%. Em 2001, 39,4\% das mães trabalhavam e, em 2005, 42,9\%. Quanto à presença de companheiro, 35,9 e 54,3\% não o referiam em 2001 e 2005, respectivamente. A escolaridade passou de $37,8 \%$ de mães com até sete anos de estudo para $25,7 \%$, com aumento das que estudaram entre oito e 11 anos e 12 anos ou mais. O comparecimento a mais de seis consultas no pré-natal passou de 74,4 para 86,6\%. Houve aumento de cesáreas $(54,9$ para $60,3 \%)$ e de prematuridade $(7,1$ para $8,9 \%)$. Não houve alteração no perfil de peso ao nascimento.

Conclusões: Verificou-se queda da paridade nos DS com piores ICV e no percentual de mães adolescentes. Elevou-se o número de trabalhadoras, mulheres sem companheiro, escolaridade e frequência ao pré-natal.

Palavras-chave: declaração de nascimento; saúde materno-infantil; nascimento vivo.

\section{ABSTRACT}

Objective: To compare data related to prenatal care, deliveries and newborns from Campinas, Brazil, in 2001 and 2005 .

Methods: This cross-sectional study analyzed 13,656 Live Birth Certificates from 2005, comparing them to of 2001. The analyzed variables were: place of birth and dwelling, maternal age, marital status, schooling, number of births, number of prenatal consultations, mode of delivery, length of pregnancy and birthweight. Association between variables was evaluated by the chi-square test, being significant $p<0.05$.

Results: In 2005, there was a decrease in the number of newborns from Health Districts with worse living conditions in comparison to 2001. The rate of teenage pregnancies varied from $17.7 \%$ in 2001 to $14.7 \%$ in 2005. Working mothers were $42.9 \%$ of the sample in 2005 and $39.4 \%$ in 2001. In 2005, single mothers were $54.3 \%$ compared to $35.9 \%$ in 2001 . Mothers with seven or less years of schooling were $37.8 \%$ in 2001, compared to $25.7 \%$ in 2005 . In $2005,86.6 \%$ of mothers had more than six prenatal visits compared to $74.4 \%$ in 2001 . There was an increase in the rates of cesarean section (from $54.9 \%$ in 2001 to $60.3 \%$ in 2005) and prematurity (from $7.1 \%$ in 2001 to $8.9 \%$ in 2005). No significant changes in birthweight were observed.

Conclusions: This study shows a decrease in the rate of births in the Health Districts with worse conditions of living and in teenage pregnancies. Rates of working

Endereço para correspondência:

Maria Ângela R. de G. M. Antonio

Avenida Modesto Fernandes, 354 (3) - Barão Geraldo

CEP 13084-190 - Campinas/SP

E-mail: anze@fcm.unicamp.br

Conflito de interesse: nada a declarar

Recebido em: 14/7/2009

Aprovado em: 26/10/2009 
mothers, single mothers, years of schooling, number of prenatal consultations increased.

Key-words: birth certificate; maternal and child health; live birth.

\section{Introdução}

Em 1990, o Ministério da Saúde implantou o Sistema de Informações sobre Nascidos Vivos (Sinasc). Para a obtenção dos dados, foi elaborado um documento básico, a Declaração de Nascido Vivo (DNV), por meio da qual se podem obter informações sobre o nascimento, o local de ocorrência, a mãe, a gestação, o parto e o recém-nascido $(\mathrm{RN})$.

Em Campinas (SP), o sistema foi implantado em 1991 e, desde 1994, está sob responsabilidade da Secretaria Municipal de Saúde. Vem sendo utilizado para diversas atividades, como: seleção de nascidos vivos de risco para seguimento, avaliação de cobertura vacinal, estimativa populacional, cálculo de coeficientes de mortalidade infantil por área de abrangência de Centro de Saúde (CS), município ou região, banco de dados de doentes e portadores de hemoglobinopatias, localização e notificação para as unidades de exames de triagem neonatal alterados. Além disso, alguns CS elegem critérios próprios para a vigilância de recém-nascidos e mães, utilizando os dados das $\mathrm{DNV}^{(1)}$.

A partir de 2001, ocorreram mudanças na atenção à saúde em Campinas. Com a implantação do modelo de Saúde da Família do Sistema Único de Saúde (SUS), constituíram-se equipes locais de referência, Núcleos de Saúde Coletiva e equipes de apoio matricial ${ }^{(2)}$. Em 2005, a rede era organizada em cinco Distritos de Saúde (DS) que abrangiam todo o município e composta por $47 \mathrm{CSs}$, com quatro a seis equipes locais cada, 13 módulos de Saúde da Família e serviços de nível secundário e terciário ${ }^{(3)}$.

Em 2001, realizou-se um estudo das 14.444 DNVs de Campinas com o objetivo de conhecer as características sociodemográficas das mães, suas gestações, partos e neonatos ${ }^{(4)}$ e contribuir para o planejamento e propostas de intervenções gerais para a população do município e, específicas, para o grupo materno-infantil. Encontrou-se alto percentual de gravidez na adolescência, parto cesáreo e peso inadequado ao nascimento, sobretudo nas regiões com piores índices de condição de vida ${ }^{(5,6)}$. Assim, este trabalho teve como objetivo comparar os dados pré-natais e dos partos das mulheres de Campinas e de seus recém-nascidos entre os anos 2001 e 2005 .

\section{Método}

O presente estudo transversal utilizou dados das 13.656 DNVs do Sinasc, referentes aos partos de mulheres residentes em Campinas, ocorridos no próprio município em 2005. Analisaram-se as características das mães e dos RNs por meio de variáveis sociodemográficas maternas (local de moradia e de parto, idade, estado civil, escolaridade, ocupação, número de filhos vivos), variáveis relacionadas à gestação (número de consultas de pré-natal e duração da gestação), ao parto e ao $\mathrm{RN}$ (tipo de parto e peso ao nascimento). Os resultados foram comparados aos obtidos em 2001.

Para avaliar a associação entre as variáveis, utilizou-se o teste de qui-quadrado. Para a análise dos dados, empregou-se o programa SPSS ${ }^{\circledR}$ (SPSS Inc. Chicago, IL USA) e, na tomada de decisão, adotou-se o nível de significância de $5 \%$ (7,8).

Este trabalho foi aprovado pelo Comitê de Ética Em Pesquisa da Faculdade de Ciências Médicas da Unicamp.

\section{Resultados}

Os resultados do ano de 2005, quando comparados aos de 2001, mostraram melhora na qualidade do preenchimento em algumas variáveis, especialmente escolaridade materna, duração da gestação e número de consultas no pré-natal (Tabela 1). Assim como em 2001, a maioria dos partos ocorreu em hospitais, havendo também diferença significativa em relação ao local de moradia, com diminuição do número de nascidos vivos nos DS noroeste e sudoeste (Tabela 2).

Quando comparado a 2001, em 2005 observou-se uma diminuição na porcentagem de mães adolescentes (de 17,7 para $14,7 \% ; p<0,001)$; aumento no número de mães com trabalho fora do lar (de 39,4 para 42,9\%; $p<0,001$ ); aumento no número de mães sem companheiro (de 35,9 para 54,3\%; $p<0,001)$; melhora do nível educacional materno, com redução na porcentagem de mães com sete ou menos anos de escolaridade (de 37,8 para 25,7\%) e aumento dos demais estratos $(p<0,001)$ (Tabela 2). Observou-se um aumento no número de mães com até um filho vivo (de 76,2 para $80,1 \%$; $p<0,001$ ) (Tabela 3).

Verificou-se maior porcentagem de mães que tiveram sete ou mais consultas de pré-natal (de 74,4 para 86,6\%; $p<0,001$ ), o mesmo ocorrendo quanto ao parto cesáreo (de $54,9$ para $60,3 \% ; p<0,001)$ e à prematuridade (de 7,1 para $8,9 \% ; p<0,001)$. Não se observou diferença quanto ao baixo peso ao nascer (de 9,1 para 9,6\%; $p=0,167$ ) (Tabela 4). 


\section{Discussão}

A cidade de Campinas (SP) é a terceira maior cidade do Estado, tem o oitavo Índice de Desenvolvimento Humano (IDH) do Estado e uma população estimada de 1.044.454 habitantes em 2005, dos quais 98,4\% residiam em área urbana $^{(9)}$.

Quase a totalidade dos nascimentos de 2001 e 2005 aconteceu em hospitais ou outros estabelecimentos de saúde $(99,9 \%)$, presumindo-se que a rede hospitalar do município ofereça leitos de obstetrícia em número suficiente.

Quanto ao local de moradia, não se verificaram, no período estudado, diferenças na proporção de nascidos vivos entre os distritos com piores e com melhores condições de vida (ICV), ao contrário do que se observa em outros locais do país com população em condições financeiras mais desfavoráveis em maior proporção, piores condições de saneamento e pior nível de escolaridade da populaçãa ${ }^{(10)}$.

Nos dois anos analisados, a maior concentração de nascimentos ocorreu no grupo de mulheres de 20 a 34 anos. Esse resultado, que se mantém desde 1994, é semelhante ao do estado $(71,8 \%)$ e ao da região metropolitana de Campinas $(73,1 \%)$ em 2005 , mas está acima da média brasileira (69\%), que apresenta uma população de mães adolescentes maior do que a do município ${ }^{(10)}$.

No grupo de mulheres com mais de 35 anos, observouse um pequeno aumento na proporção de nascimentos em relação a 2001. De acordo com o Censo 2000, à medida que aumentam os anos de estudo, o padrão da fecundidade tornase mais tardio ${ }^{(11)}$. Nos Estados Unidos, em 2005, também houve um aumento de fecundidade entre as mulheres com 30 anos ou mais, ao contrário das outras faixas etárias ${ }^{(12)}$. Na cidade de São Paulo, Martins e Almeida observaram que um número maior de mulheres optam por ter filhos mais tardiamente em virtude de seus interesses por maior escolarização e profissionalização ${ }^{(13)}$.

Tabela 1 - Distribuição da frequência de falta de informação das principais variáveis das Declarações de Nascidos Vivos, Campinas, SP

\begin{tabular}{lrrrl}
\hline \multirow{2}{*}{ Variáveis } & \multicolumn{2}{c}{$\mathbf{2 0 0 5}$} & \multicolumn{2}{c}{$\mathbf{2 0 0 1}$} \\
\cline { 2 - 5 } & $\mathbf{n}$ & $\mathbf{\%}$ & $\mathbf{n}$ & $\mathbf{\%}$ \\
\hline Idade da mãe & 5 & 0,04 & 3 & 0,02 \\
Escolaridade da mãe & 60 & 0,44 & 174 & 1,2 \\
Situação conjugal & 62 & 0,45 & 36 & 0,25 \\
Ocupação & 37 & 0,27 & 19 & 0,13 \\
Número de filhos vivos & 21 & 0,15 & 9 & 0,06 \\
Duração da gestação & 71 & 0,52 & 113 & 0,78 \\
Número de consultas & 142 & 1,04 & 1.356 & 9,39 \\
Tipo de parto & 9 & 0,07 & 4 & 0,03 \\
Peso de nascimento & 4 & 0,03 & 1 & 0,01 \\
Local de ocorrência & 0 & 0 & 0 & 0 \\
Local de moradia & 1 & 0,01 & 0 & 0 \\
\hline
\end{tabular}

Fonte: SINASC, janeiro a dezembro, 2001 e 2005, Campinas, SP, Brasil.

Tabela 2 - Distribuição dos nascidos vivos segundo o local de ocorrência de nascimento e Distrito de Saúde, Campinas, SP

\begin{tabular}{|c|c|c|c|c|c|}
\hline & \multicolumn{2}{|c|}{2005} & \multicolumn{2}{|c|}{2001} & \multirow{2}{*}{ Valor de $p$} \\
\hline & $\mathbf{n}$ & $(\%)$ & $\mathbf{n}$ & $(\%)$ & \\
\hline \multicolumn{6}{|l|}{ Local de ocorrência } \\
\hline Hospital & 13.643 & 99,9 & 14.339 & 99,3 & $<0,001$ \\
\hline Outros estabelecimentos & 1 & 0 & 51 & 0,3 & \\
\hline Domicílio & 9 & 0,1 & 38 & 0,3 & \\
\hline Outros & 3 & 0 & 16 & 0,1 & \\
\hline \multicolumn{6}{|l|}{ Local de moradia } \\
\hline DS Norte & 2.349 & 17,2 & 2.365 & 16,4 & 0,008 \\
\hline DS Sul & 3.518 & 25,8 & 3.683 & 25,5 & \\
\hline DS Leste & 2.497 & 18,3 & 2.508 & 17,4 & \\
\hline DS Noroeste & 2.282 & 16,7 & 2.554 & 17,6 & \\
\hline DS Sudoeste & 3.009 & 22 & 3.333 & 23,1 & \\
\hline
\end{tabular}

DS: Distrito de Saúde; Fonte: Sinasc. janeiro a dezembro, 2001 e 2005: Campinas, SP, Brasil. 
Tabela 3 - Distribuição das mães segundo as características sociodemográficas

\begin{tabular}{|c|c|c|c|c|c|}
\hline & \multicolumn{2}{|c|}{2005} & \multicolumn{2}{|c|}{2001} & \multirow{2}{*}{ Valor de $p$} \\
\hline & $\mathbf{n}$ & $(\%)$ & $\mathbf{n}$ & $(\%)$ & \\
\hline \multicolumn{6}{|l|}{ Idade } \\
\hline$<20$ & 2.010 & 14,7 & 2.563 & 17,7 & $<0,001$ \\
\hline $20-35$ & 9.912 & 72,6 & 10.365 & 71,8 & \\
\hline$\geq 35$ & 1.729 & 12,7 & 1.512 & 10,5 & \\
\hline \multicolumn{6}{|l|}{ Situação conjugal } \\
\hline Com companheiro & 6.215 & 45,7 & 9.242 & 64,1 & $<0,001$ \\
\hline Sem companheiro & 7.379 & 54,3 & 5.165 & 35,9 & \\
\hline \multicolumn{6}{|l|}{ Escolaridade } \\
\hline$<7$ & 3.498 & 25,7 & 5.389 & 37,8 & $<0,001$ \\
\hline $8-11$ & 7.693 & 56,6 & 6.722 & 47,1 & \\
\hline$\geq 12$ & 2.405 & 17,7 & 2.158 & 15,1 & \\
\hline \multicolumn{6}{|l|}{ Ocupação } \\
\hline Com & 5.844 & 42,9 & 5.688 & 39,4 & $<0,001$ \\
\hline Sem & 7.775 & 57,1 & 8.736 & 60,6 & \\
\hline \multicolumn{6}{|l|}{ Número de filhos vivos } \\
\hline 0 & 6.666 & 48,9 & 6.584 & 45,6 & $<0,001$ \\
\hline 1 & 4.252 & 31,2 & 4.417 & 30,6 & \\
\hline 2 & 1.645 & 12,1 & 1.938 & 13,4 & \\
\hline$\geq 3$ & 1.072 & 7,9 & 1.495 & 10,4 & \\
\hline
\end{tabular}

Fonte: Sinasc, janeiro a dezembro, 2001 e 2005: Campinas, SP, Brasil.

Tabela 4 - Distribuição das mães segundo as características da gestação, do parto e do recém-nascido

\begin{tabular}{|c|c|c|c|c|c|}
\hline & \multicolumn{2}{|c|}{2005} & \multicolumn{2}{|c|}{2001} & \multirow{2}{*}{ Valor de $p$} \\
\hline & $\mathbf{n}$ & $(\%)$ & $\mathbf{n}$ & $(\%)$ & \\
\hline \multicolumn{6}{|c|}{ Consultas de pré-natal } \\
\hline$<7$ & 1.807 & 13,4 & 3.353 & 25,6 & $<0,001$ \\
\hline$\geq 7$ & 11.707 & 86,6 & 9.734 & 74,4 & \\
\hline \multicolumn{6}{|c|}{ Duração da gestação } \\
\hline$<37$ semanas & 1.205 & 8,9 & 1.018 & 7,1 & $<0,001$ \\
\hline$\geq 37$ semanas & 12.380 & 91,1 & 13.312 & 92,9 & \\
\hline \multicolumn{6}{|l|}{ Parto } \\
\hline Vaginal & 5.423 & 39,7 & 6.515 & 45,1 & $<0,001$ \\
\hline Cesáreo & 8.224 & 60,3 & 7.924 & 54,9 & \\
\hline \multicolumn{6}{|c|}{ Peso de nascimento (g) } \\
\hline$<2500$ & 1.309 & 9,6 & 1315 & 9,1 & 0,167 \\
\hline $2500-3000$ & 3.408 & 25 & 3719 & 25,7 & \\
\hline$\geq 3000$ & 8.935 & 65,4 & 9408 & 65,1 & \\
\hline
\end{tabular}

Fonte: CII: Sinasc, janeiro a dezembro, 2001 e 2005: Campinas, SP, Brasil. 
Em relação aos partos das adolescentes, observou-se uma redução entre 2005 e 2001. Este resultado é semelhante ao da região, mas menor do que o do estado de São Paulo $(17 \%)$ e do Brasil (21,8\%). No entanto, é muito alto quando comparado aos países industrializados, cujas taxas máximas estão em torno de 4 a $5 \%{ }^{(12)}$. A preocupação com a gravidez nesta faixa etária decorre dos riscos biológicos e psicossociais a que estas mulheres estão expostas e ao impacto futuro sobre seus filhos. A interrupção da escolarização materna e da formação profissional em decorrência da gravidez dificulta a inserção no mercado de trabalho e ajuda a perpetuar a pobreza, com consequentes riscos sociais para estas mulheres e seus dependentes ${ }^{(14)}$.

Em 2005, o percentual de mulheres que tiveram filhos e que referiram trabalhar foi maior do que em 2001 e superior à média feminina brasileira em $2000(39,1 \%)^{(15)}$. Embora seja uma mudança expressiva, esse índice ainda é menor que o encontrado para os homens brasileiros, dos quais $61,1 \%$ encontram-se inseridos no mercado de trabalho. Isto pode ser decorrente das responsabilidades no cuidado com a casa e com a criação dos filhos, ainda centralizadas nas mulheres.

Quanto à escolaridade materna, tanto em 2005 como em 2001, os resultados mostraram uma situação privilegiada em relação a outras localidades do país, havendo ainda uma melhora na proporção de mulheres com mais anos de estudo e também aumento no percentual das que estudaram até o nível superior ${ }^{(10)}$. A baixa escolaridade das mães está relacionada a desfechos negativos para a sua própria saúde e a de seus filhos, tais como maior paridade, pré-natal inadequado, maior risco de mortalidade materna e infantil, risco elevado de baixo peso ao nascer, crescimento insatisfatório das crianças e imunização incorreta, entre outros ${ }^{(11,16-19)}$.

Os resultados sobre a situação conjugal mostraram que o percentual de mulheres sem companheiro aumentou entre 2001 e 2005 e foi discordante do descrito pelo IBGE para o Estado de São Paulo, onde apenas 16\% das famílias eram compostas por mulheres responsáveis pelos domicílios, sem cônjuge e com filhos ${ }^{(11)}$. Este fato pode ser explicado em parte pela possibilidade de haver mães, principalmente as mais jovens, que não têm companheiro, mas que residem no domicílio de sua família de origem, cujo responsável é outro morador. Por outro lado, também pode estar relacionada à falta de opção para o registro na DNV das mulheres com companheiro em união consensual, situação não prevista no modelo da DNV atual, pois os itens para a anotação desta variável se referem ao estado civil (solteira, casada, viúva e separada judicialmente).
Entre os nascimentos, observou-se maior proporção entre as mulheres que não tinham filhos ou que tinham somente um. Este resultado acompanha a tendência de queda da fecundidade apontada pelo Censo 2000 para o país, para as grandes regiões e para os Estados: a taxa de fecundidade total apresentou uma redução de $57 \%$ de 1970 a 2000, passando de 5,8 para 2,3 por mulher ${ }^{(10)}$. Em Campinas, no ano de 2000, essa taxa era de 2,1, uma das menores do país ${ }^{(20)}$.

Quanto ao número de consultas de pré-natal, pôde-se observar, em 2001, que a maioria das mulheres realizou sete ou mais consultas e, em 2005, esse percentual aumentou significativamente. A cobertura de pré-natal na cidade vem apresentando melhora expressiva no transcorrer dos anos e passou de 38,9\% gestantes com menos de sete consultas, em 1994, para 25,6\% em 2001 e para 13,4\% em 2005 (3).

$\mathrm{O}$ aumento de partos prematuros entre 2001 e 2005 confirma a tendência observada no decorrer dos anos, passando de 5,9\% em 1997 para 7,1\% em 2001 e 8,9\% em $2005^{(10)}$. Estudo realizado em uma maternidade da cidade detectou a mesma tendência entre 1971 e 1995 e supôs ser este fato decorrente do aumento das gestações na adolescência e da interrupção precoce da gravidez por cesárea ${ }^{(21)}$. Em Pelotas (RS), o percentual de neonatos pré-termo passou de 5,6 em 1982, para 7,5 em 1993 e, em Ribeirão Preto (SP), de 7,6 em 1978-1979 para 13,6 em 1994 ${ }^{(22)}$. Em diversos países desenvolvidos, há referência a essa mesma tendência, acarretada, provavelmente, pelas intervenções obstétricas, pela reprodução assistida e pelo decorrente aumento de partos múltiplos ${ }^{(23,24)}$.

Em relação aos partos cesáreos, houve um aumento entre 2005 e 2001, sendo realizados com maior frequência do que os partos por via vaginal. Esta tendência de aumento também foi observada nos Estados Unidos em 2006, onde $31,1 \%$ dos partos foram cirúrgicos. Apesar de a Organização Mundial da Saúde (OMS) recomendar taxas menores que $15 \%$, observa-se, desde o início da década de 1970, uma tendência mundial de aumento deste tipo de parto. Atualmente, as taxas são bastante variáveis nos diversos países, sendo que não ultrapassam $15 \%$ no Japão, na Holanda e nos países escandinavos. A França, em 1995, teve 19,5\% de partos cesáreos, a Espanha, 23\% e a Austrália, 19,5\%. Em 2000, o Reino Unido apresentou porcentagem de 21,5\% e, em 2001, o Canadá apresentou proporção de $21 \%{ }^{(25,26)}$. Na América Latina, estas taxas mostraram-se muito elevadas em diversos países e somente sete dos 19 países apresentaram taxas menores que $15 \%$; no restante houve uma variação de $16,8 \%$ na Colômbia a 40,0\% no México ${ }^{(27)}$. 
O Brasil é um dos países do mundo com maior ocorrência de cesarianas. Vem apresentando um aumento significativo no decorrer dos anos, passando de 14,6\%, no início da década de 1970, para 31,0\%, na década de 1980. Em 1995, esta proporção foi para $35,5 \%$ e se mantém neste patamar, com pequenas variações. Em 2001, a taxa média foi de 38,1\%, variando de 17,4\%, no Amapá, a 49,4\%, no Rio de Janeiro. O estado de São Paulo teve, em 2001, uma das maiores porcentagens do país $(49,1 \%)$ e a região de Campinas, uma das maiores do estado $(51,8 \%)^{(10,28)}$ (percentuais maiores do que o recomendado pela OMS). É indiscutível a contribuição deste procedimento para uma melhor assistência à saúde, no entanto, sua indicação deve ser criteriosa, pois os riscos adicionais para a mãe e para a criança são frequentemente relatados na literatura ${ }^{(25,27,28)}$.

O peso ao nascer é considerado um bom indicador do nível de saúde das populações. Neste estudo, verificou-se que a maioria das crianças, tanto em 2001 como em 2005, nasceram com peso adequado. Sua distribuição pouco se modificou desde $1995^{(29)}$, o mesmo sendo observado em outras localidades brasileiras: em São Paulo em 1998 (3157g), em Ribeirão Preto (SP) em 1994 (3115g) e em São Luís (MA) em 1997 e 1998 (3142g $)^{(30-32)}$. Esse resultado não é ideal, pois, segundo a recomendação da OMS, a proporção de nascidos com peso adequado deve ser de pelo menos $85 \%$, e o peso médio ao nascimento deve estar entre 3400 e $3500 \mathrm{~g}^{(21,31)}$.

As taxas de baixo peso ao nascer são muito variáveis nas diversas regiões do mundo, com evidentes desvantagens para os países menos desenvolvidos, pois estão associadas às más condições socioeconômicas. $\mathrm{O}$ baixo peso ao nascer tem sido objeto de vários estudos em virtude de participação importante na mortalidade e morbidade infantis e nos riscos para a saúde na vida adulta ${ }^{(23)}$. Assim, em países desenvolvidos da Europa, a taxa de baixo peso ao nascer varia entre 4 e $5 \%{ }^{(33)}$. Nos Estados Unidos, essa taxa é de $7,6 \%$, porém com variação de $13,1 \%$ na população negra a 4,8\%, na branca. As maiores proporções são as da Índia $(33,0 \%)$ e de Bangladesh (50,0\%). Na América Latina e Caribe, o valor médio é de 9,0\% e, no Brasil em 2001, a média de baixo peso ao nascer foi de $7,9 \%$, sendo a maior porcentagem no estado do Rio de Janeiro $(9,4 \%)$ e a menor em Roraima $(5,0 \%)^{(33)}$. Em geral, nos países desenvolvidos, essas taxas são principalmente decorrentes de partos prematuros, observados em dois terços dos nascimentos de crianças com baixo peso ao nascer. Nos países em desenvolvimento, o BPN decorre, na maioria das vezes, da restrição de crescimento intrauterino.
Neste estudo, a proporção de $\mathrm{RN}$ com baixo peso ao nascer, 9,1 e 9,6\% em 2001 e 2005, tem sido semelhante desde 1995, o mesmo ocorrendo na região, no Estado e em outras cidades como Ribeirão Preto (SP) (9,4\%), Porto Alegre (RS), (9,8\%), Pelotas (RS) (9,9\%) e com a média da América Latina ${ }^{(10)}$. No entanto, esta proporção é maior que o dobro da encontrada em países desenvolvidos, fato preocupante, pois as crianças nascidas com menos de $2500 \mathrm{~g}$ têm risco aumentado de morte no primeiro ano de vida, risco de desenvolverem enfermidades infecciosas e respiratórias e de evoluírem com atraso de crescimento e desenvolvimento. Alguns estudos sugerem que essas crianças podem, no futuro, apresentar doenças cardíacas, acidente vascular cerebral, hipertensão arterial, diabetes tipo 2, hiperlipidemia e obesidade ${ }^{(23)}$.

Relevante também é o número de crianças que nasceram com peso insuficiente (entre 2500 a 2999g), visto que estudos mostram risco de morbidade mais elevado do que para as nascidas com peso adequado ${ }^{(34,35)}$. Em Campinas, esta proporção se mantém inalterada desde os anos $1990^{(6,21)}$. Em 2001, para o Brasil, estado de São Paulo e região de Campinas, essas distribuições são semelhantes $(22,3 \%, 25,1 \%$ e $24,7 \%$, respectivamente). Observa-se a mesma tendência em diversos locais do país com condições de vida e de saúde semelhantes às desse município, como Porto Alegre (23,8\%), Pelotas $(23,5 \%)$, Rio de Janeiro (23,6\%) e Ribeirão Preto $(24,2 \%)^{(10)}$.

As limitações deste estudo são decorrentes da utilização de bancos de dados secundários, uma vez que nem todas as variáveis que determinam alguns desfechos estão contidas no documento de coleta. Não foi possível identificar os partos ocorridos na rede de saúde pública ou privada, pois a configuração da DNV não permite tal discriminação. Com relação à situação conjugal, a inclusão da opção "união consensual" seria necessária para que este dado pudesse refletir a real situação. O conhecimento sobre a presença do companheiro é importante, pois alguns trabalhos mostram que a ausência do pai no domicílio relaciona-se à falta de adesão ao pré-natal de gestantes adolescentes e adultas, mães de crianças com baixo peso ao nascer ${ }^{(21,30)}$.

Verificou-se melhora da maioria das variáveis estudadas entre 2005 e 2001, destacando-se queda de paridade nos DS de pior condição de vida, diminuição de mães adolescentes, aumento da ocupação e de anos de estudo e maior comparecimento ao pré-natal. $\mathrm{O}$ peso ao nascer manteve-se semelhante. Entretanto, encontraram-se mais mulheres sem companheiro, aumento de prematuros e de 
cesarianas e manutenção da tendência de redução da taxa de fecundidade.

As mudanças do modelo de atenção à saúde podem ter contribuído para a melhoria de alguns desses indicadores. No entanto, deve-se prestar atenção especial ao percentual de partos cesáreos, de modo que a assistência à gestante seja realizada dentro de critérios técnicos, proporcionando maior segurança e menor índice de complicações.

\section{Referências bibliográficas}

1. Campinas SM. Relatório SINASC - 1998 a 2000. Brasília: Ministério da Saúde; 2000.

2. Nascimento EP, Correa CR, Nozawa MR. The county of Campinas and the county health secretary office organization. Rev Cienc Med 2007;16:161-73.

3. Campinas - Secretaria Municipal de Saúde [homepage on the Internet]. Unidades - Distritos de Saúde [cited 2008 Jun 10]. Available from: http://www. campinas.sp.gov.br/saude.

4. Carniel EF. Caracterização dos recém-nascidos e de suas mães, a partir das declarações de nascidos vivos de Campinas (SP), no ano de 2001 [tese de mestrado]. Campinas (SP): FCM-Unicamp; 2005.

5. Carniel EF, Zanolli ML, Almeida CA, Morcillo AM. Characteristics of adolescent mothers and their newborns and risk factors associated to pregnancy in Campinas, SP, Brazil. Rev Bras Saude Matern Infant 2006;6:419-26.

6. Antonio MA, Zanolli ML, Carniel EF, Morcillo AM. Fatores associados ao peso insuficiente ao nascimento. Rev Assoc Med Bras 2009;55:153-7.

7. Chan YH. Biostatistics 103: qualitative data-tests of independence. Singapore Med J 2003;44:498-503.

8. Zar J. Biostatistical analysis. $2^{\text {nd }}$ ed. Englewood Cliffs: Prentice-Hall; 1984.

9. Campinas - Secretaria de Planejamento e Desenvolvimento Urbano [homepage on the Internet]. Perfil Municipal [cited 2008 Jun 10]. Available from: http://www.campinas.sp.gov.br/seplama/censo2000/portc2000.htm.

10. Brasil - Ministério da Saúde. DATASUS [homepage na Internet]. Mortalidade e Nascidos Vivos: nascidos vivos desde 1994 [cited 2008 Jun 10]. Available from: http://tabnet.datasus.gov.br/cgi/deftohtm.exe?sinasc/cnv/nvuf.def

11. Brasil - IBGE. Censo demográfico 2000: famílias e domicílios: resultados da amostra. Rio de Janeiro: IBGE; 2003.

12. Martin JA, Hamilton BE, Sutton PD, Ventura SJ, Menacker F, Kirmeyer S et al. Births: final data for 2005. Natl Vital Stat Rep 2007;56:1-103.

13. Martins CM, Almeida MF. Fertility and its intra-urban differentials of human development, Brazil, 1997. Rev Saude Publica 2001;35:421-7.

14. Aquino EM, Heilborn ML, Knaut D, Bozon M, Almeida MC, Araújo $\mathrm{J}$ et al. Adolescence and reproduction in Brazil: the heterogeneity of social profiles. Cad Saude Publica 2003;19(Suppl 2):S377-88.

15. Brasil - IBGE. Censo demográfico 2000: trabalho e rendimento - resultados da amostra. Rio de Janeiro: IBGE; 2003.

16. Antonio MA, Morcillo AM, Piedrabuena AE, Carniel EF. Growth analysis of 566 children from the fourteen day care centers of Paulínia (São Paulo, Brazil), with ages ranging from 3 months to 3 years. J Pediatr (Rio J) 1996;72:245-50.

17. Silva AA, Gomes UA, Tonial SR, Silva RA. Vaccination coverage and some risk factors for non-vaccination in an urban area of Northeastern Brazil, 1994. Rev Saude Publica 1999;33:147-56.
Por meio de instrumentos do cotidiano, pode-se nortear a implementação de políticas de saúde materno-infantil e avaliar os impactos dessas intervenções. Esforços devem ser feitos para a manutenção da alta cobertura do Sinasc no município e para que se procedam às avaliações sistematizadas, sendo possível, com isso, identificar as falhas existentes e aperfeiçoar o sistema para que os estudos possam ser feitos com base em dados confiáveis.

18. Moraes Neto OL, Barros MB. Risk factors for neonatal and post-neonata mortality in the Central-West region of Brazil: linked use of life birth and infant death records. Cad Saude Publica 2000;16:477-85.

19. Haidar FH, Oliveira UF, Nascimento LF. Escolaridade materna: correlação com os indicadores obstétricos. Cad Saude Publica 2001;17:1025-9.

20. SEADE [homepage on the Internet]. Estatísticas vitais do Estado de São Paulo - resenha mensal. ano 4(3), junho, 2003 [cited 2008 Mai 02]. Available from: http://www.seade.gov.brs

21. Mariotoni GG. Tendência secular do peso ao nascer em Campinas, 1971-1975 [tese de doutorado]. Campinas (SP): FCM-Unicamp; 1998.

22. Aragão VM, Silva AA, Aragão LF, Barbieri MA, Bettiol H, Coimbra LC et al. Risk factors for preterms births in São Luis, Maranhão, Brazil. Cad Saude Publica 2004;20:57-63.

23. Kramer MS. Determinants of low birth weight: methodological assessment and meta-analysis. Bull World Health Organ 1987;65:663-737.

24. Tucker J, McGuire W. Epidemiology of preterm birth. BMJ 2004;329:675-8.

25. Federação Brasileira de Ginecologia e Obstetrícia. Manual de orientação. São Paulo: Febrasgo; 2002.

26. Dobson R. Caesarean section rate in England and Wales hits 21. BMJ 2001;323:951.

27. Belizán JM, Althabe F, Barros FC, Alexander S. Rates and implications of caesarean sections in Latin America: ecological study. BMJ 1999;319:1397-402.

28. Rattner D. Sobre a hipótese de estabilização das taxas de cesárea no estado de São Paulo, Brasil. Rev Saude Publica 1996;30:19-33.

29. Bicalho-Mariotoni GG, Barros Filho AA. Nascer em Campinas: análise de dados do SINASC, 1995. Rev Paul Pediatr 1997;15:24-30.

30. Barbieri MA, Silva AA, Bettiol H, Gomes UA. Risk factors for the increasing trend in low birth weight among live births born by vaginal delivery, Brazil. Rev Saude Publica 2000;34:596-602.

31. Monteiro CA, Benício MH, Ortiz LP. Secular trends in birth weight in S. Paulo city, Brazil (1976-1998). Rev Saude Publica 2000;34 (Suppl 6):26-40.

32. Silva AA, Coimbra LC, Silva RA, Alves MT, Lamy Filho F, Lamy ZC et al. Perinatal health and mother-child health care in the municipality of São Luís, Maranhão State, Brazil. Cad Saude Publica 2001;17:1413-26.

33. UNICEF [homepage on the Internet]. The state of the world's children 2000 [cited 2008 Mai 22]. Available from: http://www.unicef.org/sowc00/.

34. Puffer RR, Serrano CV. Caracteristicas del peso al nascer. Washington, DC: Organización Panamericana de la Salud; 1988.

35. Azenha VM, Mattar MA, Cardoso VC, Barbieri MA, Del Ciampo LA, Bettiol $H$. Insufficient birth weight: factors associated in two cohorts of newborns in Ribeirão Preto, São Paulo, Brazil. Rev Paul Pediatr 2008;26:27-35. 